\title{
An Application of Stahl's Conjecture About the $k$-tuple Chromatic Numbers of Kneser Graphs
}

\author{
Svata Poljak and Fred S. Roberts*
}

\begin{abstract}
A $k$-tuple coloring of a graph $G$ assigns a set of $k$ colors to each vertex of $G$ so that if two vertices are adjacent, the corresponding sets of colors are disjoint. The $k$-tuple chromatic number of $G$ is the smallest $t$ so that there is such a $k$-tuple coloring of $G$ using $t$ colors in all. The Kneser graph $K(m, n)$ has as vertices all $n$-element subsets of the set $\{1,2, \ldots, m\}$ and an edge between two subsets iff they are disjoint. The value of the $k$-tuple chromatic number of the Kneser Graph is the subject of a 30-year-old conjecture of Saul Stahl. This paper summarizes known results about Stahl's Conjecture and applies the ideas to answer two questions of N.V.R. Mahadev about the relation between the $n$-tuple chromatic number of a graph and $n$ times the size of its largest clique.
\end{abstract}

\section{Introduction}

Graph coloring is an old subject with many important applications. Variants of graph coloring are not only important in their various applications, but they have given rise to some very interesting mathematical challenges and open questions. Our purpose in this mostly expository paper is to draw attention to a conjecture of Saul Stahl's about one variant of graph coloring, $k$-tuple coloring. Stahl's Conjecture remains one of the long-standing, though not very widely known, conjectures in graph the-

Fred S. Roberts

Department of Mathematics and DIMACS, Rutgers University, e-mail: name@email.address

* Dedication by Fred Roberts: This paper is dedicated to Peter Fishburn. His collaboration over the years has been a source of pleasure and inspiration to me. Because of our joint work on generalizations of ordinary graph colorings, it is especially appropriate to dedicate this paper to Peter. Not only is he a colleague, but I am pleased to call him a friend.

Acknowledgements: Fred Roberts thanks the National Science Foundation for its support under grant CCF-0514703 to Rutgers University. He is indebted to the late Svata Poljak for his insights and ideas that form the basis for many of the results in this paper. 
ory. We also apply a special case of the conjecture to answer two questions about $k$-tuple coloring due to N.V.R. Mahadev.

An interesting and important variant of ordinary graph coloring involves assigning a set of $k$ colors to each vertex of a graph so that the sets of colors assigned to adjacent vertices are disjoint. Such an assignment is called a $k$-tuple coloring of the graph. $k$-tuple colorings were introduced by Gilbert [6] in connection with the mobile radio frequency assignment problem (see $[15,17,18,19])$. Other applications of such multicolorings include fleet maintenance, task assignment, and traffic phasing. These are discussed in $[15,18,19]$ and elsewhere. Among the early publications on this topic are $[1,2,5,20,23]$. Given a graph $G$ and positive integer $k$, we seek the smallest number $t$ so that there is a $k$-tuple coloring of $G$ using colors from the set $\{1,2, \ldots, t\}$. This $t$ is called the $k^{\text {th }}$ multichromatic number or $k$-tuple chromatic number of $G$ and is denoted by $\chi_{k}(G)$. Of course, if $k=1, \chi_{k}(G)$ is just the ordinary chromatic number $\chi(G)$.

A homomorphism from graph $G$ to graph $H$ is a function $h$ assigning each vertex of $G$ to a vertex of $H$ so that if $x$ and $y$ are adjacent in $G$, then $h(x)$ and $h(y)$ are adjacent in $H$. It is well known that an ordinary graph coloring of a graph $G$ with $m$ colors is a homomorphism from $G$ into the complete graph $K_{m}$ of $m$ vertices. Similarly, an $n$-tuple coloring of a graph $G$ with $m$ colors is a homomorphism from $G$ into the Kneser graph $K(m, n)$. This is the graph whose vertex set consists of all $n$ element subsets of $\{1,2, \ldots, m\}$, and which has an edge between two such subsets if they are disjoint. (We assume $m \geq 2 n$, for otherwise $K(m, n)$ has no edges.) Lovász [13] computed the ordinary chromatic number $\chi(K(m, n))$ in the process of settling the famous Kneser Conjecture:

Kneser's Conjecture: If the $n$-element subsets of a $2 n+p$-element set are split into $p+1$ classes, then one of the classes will contain two disjoint $n$-element sets.

Restated, the conjecture says the following:

Kneser's Conjecture Restated: $\chi(K(2 n+p, n) \geq p+2$.

Lovász proved this conjecture by showing the following:

Theorem 1.1 (Lovász [13]): $\chi(K(m, n))=m-2 n+2, m \geq 2 n$.

This leads naturally to the question: What is $\chi_{k}(K(m, n))$ ? In [23], Stahl conjectured the following:

Stahl's Conjecture: If $k=q n-r, q \geq 1,0 \leq r<n$, then $\chi_{k}(K(m, n))=q m-2 r$.

This conjecture has remained open since 1976 and very little progress has been made on it since Stahl's original paper. Section 2 summarizes what is known about Stahl's Conjecture. We make use of Lovász' Theorem and a special case of Stahl's Conjecture in Section 3. Our purpose is to illustrate an amusing application of these two ideas and at the same time highlight Stahl's Conjecture. 
It is easy to show that $n \omega(G) \leq \chi_{n}(G) \leq n \chi(G)$, where $\omega(G)$ is the size of the largest clique of $G$. Hence, the weakly $\gamma$-perfect graphs, those for which $\chi=\omega$, have the property that $\chi_{n}(G)=n \chi(G)$. This observation led N.V.R. Mahadev [14] to ask how good the lower bound $n \omega(G)$ for $\chi_{n}(G)$ is. In particular, he asked the following questions, which we settle in Section 3.

- Question 1: If $\chi_{n}(G)=n \omega(G)$, does this imply that $\chi(G)=\omega(G)$ ?

Question 1 suggests that if $\chi(G) \neq \omega(G)$, then $\chi_{n}(G) \geq n \omega(G)+1$. Mahadev conjectured that the answer to the following question is true:

- Question 2: Is $\chi_{n}(G) \geq n \omega(G)+[\chi(G)-\omega(G)]$ ?

In Section 3, we settle these questions, using Lovász' Theorem and a special case of Stahl's Conjecture.

\section{Known Results Concerning Stahl's Conjecture}

Here we recall some known results.

Stahl [23] showed that the upper bound in his conjecture always holds:

Theorem 2.1 (Stahl ([23]): If $k=q n-r, q \geq 1,0 \leq r<n$, then $\chi_{k}(K(m, n)) \leq$ $q m-2 r$.

We will need the following result in the next section. It also gives a simple proof that Stahl's Conjecture holds if $1 \leq k \leq n$.

Theorem 2.2 (Stahl ([23]): If $G$ has an edge and $n>1$, then $\chi_{n}(G) \geq 2+\chi_{n-1}(G)$.

Theorem 2.3 (Stahl ([23]): If $1 \leq k \leq n$, then $\chi_{k}(K(m, n))=m-2(n-k)$.

Proof. The upper bound follows by Theorem 2.1. The lower bound follows by repeated use of Theorem 2.2 and Theorem 1.1.

Corollary 2.4 (Stahl ([23]): Stahl's Conjecture holds if $1 \leq k \leq n$.

Theorem 2.5 (Stahl ([23]): $\chi_{u n}(K(m, n))=u m, u>0$.

Corollary 2.6 (Stahl ([23]): Stahl's Conjecture holds if $k=u n, u>0$.

Theorem 2.7 (Stahl ([23]):

$$
\chi_{k}(K(2 n+1, n))=2 k+1+\left\lfloor\frac{k-1}{n}\right\rfloor .
$$

Corollary 2.8 (Stahl ([23]): Stahl's Conjecture holds if $m=2 n+1$.

Theorem 2.9 ${ }^{2}$ Stahl's Conjecture holds for $n=2,3$.

${ }^{2}$ This was proven in [24]. According to [24], it was independently and previously proven for $n=2$ by Claude Tardif. 
Theorem 2.10 (Garey and Johnson [5]): Stahl's Conjecture holds if $n=3, k=$ $4, m \geq 6$

By using $q n+p=(q+1) n-(n-p)$, we see that Stahl's conjecture is equivalent to

If $k=q n+p, q \geq 0,0<p \leq n$, then $\chi_{k}(K(m, n))=q m+m-2 n+2 p$.

By Theorem 2.1, we know that the upper bound in Stahl's conjecture holds. By Theorems 2.2 and 2.5, the lower bound follows if

$$
\chi_{n q+1}(K(m, n)) \geq \chi_{n q}(K(m, n))+m-2 n+2,
$$

i.e., if the lower bound holds for $p=1$. As Osztényi [16] points out, it follows from a result of Stahl [24] that

$$
\chi_{n q+1}(K(m, n)) \geq \chi_{n q}(K(m, n))+m-n+2-f(n),
$$

where $f(n)=n^{2}-3 n+4$. This shows that, given $n$ and $c \in(0,1)$, we have

$$
\chi_{n q+1}(K(m, n)) \geq \chi_{n q}(K(m, n))+c[m-2 n+2]
$$

for $m$ large enough. Note that if $m \leq n^{2}-n+4$, Stahl's results in [24] imply that

$$
\chi_{n q+1}(K(m, n)) \geq \chi_{n q}(K(m, n))+2 .
$$

Ostényi [16] shows that, in fact,

$$
\chi_{n q+1}(K(m, n)) \geq \chi_{n q}(K(m, n))+3
$$

for all positive integers $n, m, q .^{3}$

There have been few other results about Stahl's conjecture over the years, though it is mentioned from time to time in the literature. Frankl and Füredi [4] discuss extremal problems on Kneser graphs and mention the Stahl Conjecture. Tardif and Zhu [25] show that if the conjecture is true, then only very few Kneser graphs are multiplicative. (A graph $K$ is called multiplicative if for any two graphs $G$ and $H$ that are not homomorphic to $K$, their categorical product or tensor product is also not homomorphic to $K$.)

\section{Answers to Mahadev's Questions}

We first show that Question 2 has an affirmative answer if $\omega=2$.

Proposition 3.1 If $\omega(G)=2$, then

${ }^{3}$ The author thanks József Ostényi for sharing an early version of his paper, in which these ideas are developed. 


$$
\chi_{n}(G) \geq n \omega(G)+[\chi(G)-\omega(G)] .
$$

Proof. Suppose $\omega(G)=2$. By Theorem 2.2,

$$
\chi_{n}(G) \geq 2(n-1)+\chi_{1}(G) .
$$

Thus, since $\omega(G)=2$ and $\chi_{1}(G)=\chi(G)$,

$$
\chi_{n}(G) \geq n \omega(G)+[\chi(G)-\omega(G)] .
$$

We observe next that the bound in Question 2 fails in general.

Proposition 3.2 There are graphs for which

$$
\chi_{n}(G)<n \omega(G)+[\chi(G)-\omega(G)] .
$$

Proof. By Theorem 2.5, we know that

$$
\chi_{n}(K(m, n))=m
$$

By Theorem 1.1,

$$
\chi(K(m, n))=m-2 n+2 .
$$

Since a clique in $K(m, n)$ consists of a disjoint collection of $n$-element subsets of $\{1,2, \ldots, m\}$, we note that

$$
\omega(K(m, n))=\left\lfloor\frac{m}{n}\right\rfloor
$$

If

$$
\chi_{n}(G) \geq n \omega(G)+[\chi(G)-\omega(G)],
$$

then by (1), (2), and (3), we have

$m=\chi_{n}(K(m, n)) \geq n \times\left\lfloor\frac{m}{n}\right\rfloor+(m-2 n+2)-\left\lfloor\frac{m}{n}\right\rfloor \geq n\left(\frac{m}{n}-1\right)+(m-2 n+2)-m / n$,

so

$$
3 n-m+\frac{m}{n}-2 \geq 0 .
$$

Certainly if $m=p n, p \geq 4, n \geq p$, then (4) fails.

We next observe that the answer to Question 1 is "no".

Proposition 3.3 There are graphs for which $\chi_{n}(G)=n \omega(G)$, but $\chi(G) \neq \omega(G)$. 
Proof. Consider the Kneser graph $K(m, 2)$ for $m$ even. By (1) and (3),

$$
\chi_{2}(K(m, 2))=m=2 \omega(K(m, 2)) .
$$

However, by (2),

$$
\chi(K(m, 2))=m-2,
$$

while by (3),

$$
\omega(K(m, 2))=m / 2,
$$

so $\chi \neq \omega$ already for $m=6$.

\section{Closing Remarks}

Several other related directions of work are of interest. Hilton, Rado, and Scott [9] define the multichromatic number (sometimes called the ultimate chromatic number) $\chi^{*}(G)$ to be $\inf _{k}\left(\chi_{k}(G) / k\right)$. Clarke and Jamison [2], Lovász [12], and Scott [20] independently showed that this is equal to $\chi_{q}(G) / q$ for some $q$. Of course, if $G$ is weakly $\gamma$-perfect, then $q=1$. Johnson, Holroyd, and Stahl [10] showed that $\chi^{*}(K(m, n))=m / n$, if $n \geq 2, m>n$. They studied the relation between the multichromatic number and star chromatic number introduced by Vince [26]. Another long-standing conjecture in graph theory is the conjecture in [10] that the star chromatic number of a Kneser graph is equal to its chromatic number. Simonyi and Tardos [21] proved this conjecture if the chromatic number is even. The star chromatic number arises by considering the set of colors $M=\{1,2, \ldots, m\}$ as residue classes modulo $m$. Thus, the distance $d(x, y)$ between two colors $x, y$ in $M$ is the distance between $x$ and $y$ around the circle of $M$ points, i.e., the minimum of $(x-y) \bmod (m)$ and $(y-x) \bmod (m)$. Assume that $m, D$ are positive integers, $G$ has at least one edge and has chromatic number at most $m$. Then an $(m, D)$-coloring of $G$ is an assignment of a color $f(a)$ to every vertex $a$ of $G$ using residue classes modulo $m$ so that the minimum $d(f(a), f(b))$ is at least $D$. We define $\eta_{m}(G)$ to be the maximum $D$ so

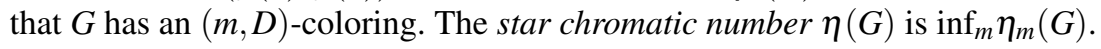

Clarke and Jamison [2], Lovász [12], and Scott [20] observed that the multichromatic number can be calculated by a linear program. This number and the $k$-tuple chromatic number are closely related to the fractional chromatic number that can also be calculated by a linear program. For an early summary of the relationships among $k$-tuple chromatic numbers, multiple chromatic numbers, fractional chromatic numbers, and their analogues for independence number, clique number, and clique covering number, see [8]. For a comprehensive summary of the literature of fractional chromatic number, see [22].

Klostermeyer and Zhang [11] showed that any planar graph $G$ with odd girth at least $10 n-7, n \geq 2$, has a homomorphism to the Kneser graph $K(2 n+1, n)$, i.e., 
$\chi_{n}(G) \leq 2 n+1$. (The case $n=1$ fails since that would say that every planar graph of odd girth at least 3 is 3-colorable. However, by Grötzsch's Theorem [7], every planar graph of odd girth at least 5 is 3 -colorable.)

It is not hard to show that for any graph $G$,

$$
\chi_{k+1}(G) \leq \chi_{k}(G)+\chi(G) \leq 2 \chi_{k}(G) .
$$

Indeed, the first part follows from the more general result in [23] that

$$
\chi_{q p+r}(G) \leq q \chi_{p}(G)+\chi_{r}(G) .
$$

Equation (5) for $G=K(m, n)$ follows directly from Theorem 2.3 if $1 \leq k \leq n$. Lovász [3] asked whether, for every $k$, there are graphs $G$ for which $\chi_{k+1}(G)>$ $(2-\varepsilon) \chi_{k}(G)$. Chvátal, Garey, and Johnson [1] showed that this was indeed the case.

It should be noted that $\chi_{n}(G)$ can be arbitrarily larger than $n \omega(G)+[\chi(G)-$ $\omega(G)$ ]. Indeed, the odd cycles $C_{2 p+1}$ illustrate this point. Stahl [23] shows that

$$
\chi_{n}\left(C_{2 p+1}\right)=2 n+1+\left\lfloor\frac{n-1}{p}\right\rfloor .
$$

However,

$$
n \omega\left(C_{2 p+1}\right)+\left[\chi\left(C_{2 p+1}-\omega\left(C_{2 p+1}\right)\right]=2 n+1 .\right.
$$

One can ask for a characterization of graphs for which

$$
\chi_{n}(G)=n \omega(G)+[\chi(G)-\omega(G)]
$$

and also for a characterization of graphs for which

$$
\chi_{n}(G)>n \omega(G)+[\chi(G)-\omega(G)]
$$

and one of graphs for which

$$
\chi_{n}(G)<n \omega(G)+[\chi(G)-\omega(G)] .
$$

These make for intriguing open questions.

\section{References}

1. Chvátal, V., Garey, M.R., and Johnson, D.S., "Two results concerning multicoloring," Annals Discrete Math., 2 (1978), 151-154.

2. Clarke, F.H., and Jamison, R.E., "Multicolorings, measures, and games on graphs," Discrete Math., 4 (1976), 241-246.

3. Erdös, P, personal communication to Chvátal, Garey, and Johnson.

4. Frankl, P., and Füredi, Z., "Extremal problems concerning Kneser graphs," J. Combin. Theory, B40 (1986), 270-284 
5. Garey, M.R., and Johnson, D.S., "The complexity of near optimal graph coloring," J. Assoc. Comput. Mach., 23 (1976), 43-49.

6. Gilbert, E.N., Unpublished technical memorandum, Bell Telephone Labs, Murray Hill, NJ, 1972.

7. Grötzsch, H., "Ein dreifarbensatz für dreikresifreie netze auf der kugel," Wissenschaftliche Zeitschrift der Martin Luther Universität Halle-Wittenburg, Math Naturwiss. Reihe, 8 (1958/1959), 109-120.

8. Hell, P., and Roberts, F.S., "Analogues of the Shannon capacity of a graph," Annals Discrete Math., 12 (1982), 155-168.

9. Hilton, A.J.W., Rado, R., and Scott, S.H., "Multicolouring graphs and hypergraphs," Nanta Mathematica, IX (1975), 152-155.

10. Johnson, A., Holroyd, F.C., and Stahl, S., "Multichromatic numbers, star chromatic numbers and Kneser graphs," J. of Graph Theory, 26 (1997), 137-145.

11. Klostermeyer, W., and Zhang, C.Q., " $n$-tuple coloring of planar graphs with large odd girth," Graphs and Combinatorics, 18 (2002), 119-132.

12. Lovász, L., "Minimax theorems for hypergraphs," in C. Berge and D. Raychaudhuri (eds.), Hypergraph Seminar, Lecture Notes in Mathematics, Vol. 411, Springer-Verlag, Berlin, 1972, 111-126.

13. Lovász, L., "Kneser's conjecture, chromatic number and homotopy," J. Combin. Theory, A25 (1978), 319-324.

14. Mahadev, N.V.R., personal communication, November 9, 1990.

15. Opsut, R.J., and Roberts, F.S., "On the fleet maintenance, mobile radio frequency, task assigment, and traffic phasing problems," in G. Chartrand, et al. (eds.), The Theory and Applications of Graphs, Wiley, New York, 1981, 479-492.

16. Ostënyi, J., "A lower bound on the multichromatic number of the Kneser graphs," preprint, Bolyai Institute, University of Szeged, 2007.

17. Roberts, F.S., Graph Theory and its Applications to Problems of Society, CBMS-NSF Monograph No. 29, Society for Industrial and Applied Mathematics, Philadelphia, 1978.

18. Roberts, F.S., "On the mobile radio frequency assignment problem and the traffic light phasing problem," Annals, NY Acad. of Sci., 319 (1979), 466-483.

19. Roberts, F.S., and Tesman, B., Applied Combinatorics, 2nd ed., Pearson Prentice Hall, Upper Saddle River, NJ, 2005.

20. Scott, S.H., Multiple Node Colorings of Finite Graphs, Ph.D. Dissertation, University of Reading, England, March 1975.

21. Simonyi, G., and Tardos, G., "Local chromatic number and the Borsuk-Ulam Theorem," http://front.math.ucdavis.edu/math.CO/0407075

22. Scheinerman, E.R., and Ullman, D.H., Fractional Graph Theory: A Rational Approach to the Theory of Graphs, Wiley, New York, 1997.

23. Stahl, S., " $n$-Tuple colorings and associated graphs," J. Combin. Theory, B20 (1976), 185203.

24. Stahl, S., "The multichromatic numbers of some Kneser graphs," Discrete Math., 185 (1998), 287-291.

25. Tardif, C., and Zhu, X., "The level of nonmultiplicativity of graphs," Discrete Math., 244 (2002), 461-471.

26. Vince, A., "Star chromatic number," J. of Graph Theory, 12 (1988), 551-559. 